\title{
Abandono no Ensino Superior: Impacto da autoeficácia na intenção de abandono ${ }^{1}$
}

\author{
Joana R. Casanova ${ }^{2}$ \\ Universidade do Minho, Braga, Portugal \\ Antonio Cervero Fernandez-Castañon \\ José Carlos Nuñez Pérez \\ Ana B. Bernardo Gutiérrez \\ Universidade de Oviedo, Oviedo, Espanha \\ Leandro S. Almeida \\ Universidade do Minho, Braga, Portugal
}

\section{Resumo}

As taxas crescentes do abandono no Ensino Superior acompanham a maior democratização do acesso, justificando o seu estudo. Buscou-se analisar o impacto de variáveis pessoais, percurso escolar, escolha do curso e perceções de autoeficácia na intenção de abandono. Tomando 1.085 estudantes do $1 .^{\circ}$ ano, aplicou-se a Escala de Autoeficácia na Formação Superior (Vieira, Polydoro, \& Guerreiro-Casanova, 2017). Recorrendo à Modelagem de Equações Estruturais, os resultados realçam o efeito das variáveis sexo, habilitação académica dos pais, média de acesso e frequência de curso de primeira opção na intenção de abandono. A autoeficácia, para além do impacto direto na intenção de abandono, mediou o impacto da média de acesso e do curso de primeira opção. Mencionam-se medidas para minorar o problema.

Palavras-chave: Ensino superior, evasão, autoeficácia

\section{Abstract: Dropout in higher education: Impact of self-efficacy in dropout intention}

The increasing rates of dropout in Higher Education follow the greater democratization of access, justifying its study. It is intended to analyze the impact of personal variables, school course, degree choice and perceptions of self-efficacy in dropout intention. The Self-efficacy Scale in Higher Education (Vieira, Polydoro, \& Guerreiro-Casanova, 2017) was applied to 1,085 first year students. Applying the Structural Equation Modeling, the results highlight the effect of the variables gender, parental academic qualification, grade point average to access higher education and frequency of first choice degree in the intention of dropout. Self-efficacy, in addition to the direct impact on the intention to dropout, measured the impact of the grade point average and the first option degree. Some recommendations to reduce the problem are mentioned.

Keywords: Higher education, dropout, self-efficacy

Resumen: Abandono en la enseñanza superior: Impacto de la autoeficacia en la intención de abandono

Las tasas crecientes de abandono en la enseñanza superior van en paralelo con una mayor democratización del acceso, lo que justifica su estudio. Se ha propuesto analizar el impacto de variables personales como trayectoria escolar, elección de la carrera y percepciones de autoeficacia en la intención de abandono. Tomando 1.085 estudiantes de primer año, se aplicó la Escala de Autoeficacia en la Formación Superior (Vieira, Polydoro, y Guerrero-Casanova, 2017). Apoyándonos en el Modelo de Ecuaciones Estructurales, en los resultados resalta el efecto de las variables sexo, preparación académica de los padres, media de acceso y frecuencia de la carrera universitaria solicitada en primera opción en la intención de abandono. La autoeficacia, además del impacto directo en la intención de abandono, sirvió para medir el impacto del promedio de acceso y de la carrera solicitada en primera opción. Se mencionan medidas para mitigar el problema.

Palabras clave: Enseñanza superior, abandono, autoeficacia

\footnotetext{
${ }^{1}$ Este trabalho é financiado pelo CIEd - Centro de Investigação em Educação, projetos UID/CED/1661/2013 e UID/CED/1661/2016, Instituto de Educação, Universidade do Minho, através de fundos nacionais da FCT/MCTES-PT. A primeira autora beneficia também de financiamento da Fundação para a Ciência e a Tecnologia através de uma Bolsa de Investigação (SFRH/BD/117902/2016).

2 Endereço para correspondência: Centro de Investigação em Educação (CIEd), Universidade do Minho, 4710-057, Braga, Portugal. E-mail: joana.casanova@gmail.com
} 
O abandono do Ensino Superior (ES) é um tema de importante análise no âmbito do Espaço Europeu de Educação Superior, pois é assumido como um indicador de qualidade das instituições universitárias (Bernardo et al., 2015). O abandono provoca, também, consequências nefastas aos estudantes a nível pessoal, familiar, social e económico (Pascarella \& Terenzini, 1991). Antes de avançar no artigo importa esclarecer a utilização do termo "abandono" no presente artigo. No Brasil, o Ministério da Educação e Cultura (1998), clarifica que a evasão do curso pode ocorrer através de (a) abandono (deixar de se matricular), (b) desistência (formalização junto da universidade), (c) transferência/reopção (mudança de curso), e, (d) exclusão por norma institucional. A evasão da instituição se refere à saída da instituição em que se matriculou e a evasão do sistema se refere à saída temporária ou definitiva do Ensino Superior. Em Portugal, havendo o paralelo destes fenômenos, não há ainda uma clarificação e uniformização da utilização das designações a nível do Ministério da Educação, constando nos documentos oficiais a referência a estudantes "não encontrados no sistema de ensino". Em termos de investigação e em consonância com os objetivos específicos de cada estudo, é mais frequente falar-se de abandono do Ensino Superior (abandono definitivo ou temporário da formação de nível superior), abandono da instituição (identificáveis pela não matrícula em anos subsequentes ou pela anulação de matrícula) e o abandono do curso (referente maioritariamente às mudanças de curso dentro da mesma instituição).

Pela sua relevância, esta problemática tem vindo a ser cada vez mais estudada e abarca um conjunto alargado de variáveis psicológicas, sociais, institucionais e econômicas. A investigação mostra que as variáveis pessoais como a motivação e os projetos vocacionais ou de carreira, variáveis sociais como o estatuto socioeconômico e a escolaridade dos pais, entre outras, são relevantes no processo de decisão de abandonar os estudos, privilegiando-se a sua interação mais do que a importância de cada uma delas isoladamente (Comissão Europeia, 2014). Basicamente, a decisão de abandonar o ES deve ser entendida como um processo de decisão vocacional dinâmico e de convergência de múltiplos fatores (Stinebrickner \& Stinebrickner, 2014), não sendo possível entendê-la como uma decisão imediata ou pontual. As vivências dos estudantes nos primeiros tempos na universidade assumem-se como determinantes na forma como estes vão conhecendo e enfrentando os desafios do novo contexto académico. Num período crítico de adaptação, estes estudantes terão de ir mobilizando recursos para enfrentarem com sucesso os desafios e conseguirem viver satisfatoriamente a experiência do ES. Quando tal não acontece, o estudante vai equacionando os custos e as dificuldades experienciadas, sejam elas financeiras ou de índole mais emocional e social, surgindo gradualmente a intenção de abandonar o ES (Stinebrickner \& Stinebrickner, 2014).

Considerando alguns dos modelos internacionais explicativos do abandono do ES (e.g. Astin, 1984; Bean, 1980; Spady, 1970; Tinto, 1975), todos eles contribuem para uma análise do abandono como um processo de desvinculação gradual, traduzido em pensamentos e intenções de abandono. Por exemplo, partindo do modelo de Tinto, Bean (1980) valoriza a socialização do estudante, assumindo que os aspetos académicos, institucionais e sociais impactam na intenção de abandonar. Por sua vez, Astin (1984) destaca o papel do esforço e da dedicação dos estudantes reforçando a importância das intenções e a natureza processual da decisão de abandono (Duque, Duque, $\&$ Suriñach, 2013). Este modelo, aliás, pode ser entendido como precursor dos modelos atuais de engagement, nas vertentes cognitiva, emocional e comportamental (Bresó, Schaufeli, \& Salanova, 2011; Fredricks, Blumenfeld, \& Paris, 2004) e, por outro lado, destaca a satisfação académica na análise da persistência e do abandono do Ensino Superior. Assumindo o abandono como processo, Bean e Metzner (1985) destacam a relevância da intenção de abandono que está relacionada com o rendimento académico, com questões académicas (e.g. hábitos de estudo), com aspetos psicológicos (e.g. autoeficácia), satisfação e com variáveis do contexto (e.g. situação financeira ou ser estudante-trabalhador). Neste sentido, a intenção de abandono impacta direta ou indiretamente na decisão final de abandonar o ES.

Em síntese, a investigação na área enumera um conjunto alargado de variáveis, como o sexo ou a idade, o ter responsabilidades familiares ou profissionais, o percurso escolar prévio, o projeto vocacional pouco desenvolvido ou inexistente, os problemas de saúde, as dificuldades econômicas, as relações interpessoais e institucionais, entre outras. A sua relevância na expressão do abandono pode diferir de contexto para contexto, validando desta forma, a necessidade de estudos contextualizados para uma avaliação correta das dificuldades especificas de cada estudante e consequente desenvolvimento de medidas de apoio (Carvalho \& Taveira, 2012).

Uma variável bastante estudada no âmbito do ES é a autoeficácia dos estudantes, mais especificamente a autoeficácia académica. A sua relevância aparece hoje reforçada em face da maior diversidade de públicos que acedem ao ES. Na sua definição, a autoeficácia é entendida como o conjunto de crenças pessoais que um indivíduo constrói com base na sua experiência de vida e que influenciam o tipo de respostas motivacionais, cognitivas e afetivas nos 
contextos de aprendizagem e realização (Bandura, 1996; Criollo, Romero, \& Fontaines-Ruiz, 2017; Polydoro \& Guerreiro-Casanova, 2010). No domínio especifico do ES, a autoeficácia académica refere-se, então, à confiança ou crença de que o estudante consegue concretizar tarefas e atingir objetivos com sucesso, podendo níveis mais elevados ou adequados de autoeficácia reduzir a probabilidade de abandono (Azzi \& Polydoro, 2007). As perceções de autoeficácia dos estudantes medeiam as vivências académicas e as suas perceções, assim como a fixação de metas e objetivos na educação superior. As perceções de autoeficácia vão também relacionar-se com o investimento (engagement) e as estratégias de enfrentamento (por exemplo, mecanismos de autorregulação) na persistência do estudante face às dificuldades, influenciando os níveis de stress, ansiedade, a satisfação e bem-estar, assim como o seu rendimento académico (Ambiel, Santos, \& Dalbosco, 2016; Casanova, Esteban, Cervero, Bernardo, \& Almeida, 2017; Nunes, 2008). A título de exemplo, os estudantes com perceções positivas de autoeficácia, apresentam níveis superiores de autorregulação no estudo e abordagens mais profundas na sua aprendizagem (Soares, Pinheiro, Manuel, \& Canavarro, 2015).

A investigação sugere que estando as perceções de autoeficácia associadas ao rendimento académico, também por essa via assume um papel mediador da persistência ou abandono (Bernardo et al., 2015). Segundo Guerreiro-Casanova e Polydoro (2011), a autoeficácia na formação superior pode ser descrita e avaliada tomando cinco dimensões: (a) a autoeficácia académica refere-se à crença do estudantes de que tem a capacidade e a competência de aprender os conteúdos e de os colocar em prática; (b) a autoeficácia na regulação da formação refere-se à perceção da capacidade de estabelecer objetivos e metas, de fazer o planeamento e de ter comportamento em que a formação e o desenvolvimento vocacional e de carreira estejam presentes; (c) a autoeficácia nas interações sociais, diz respeito à perceção de capacidade para estabelecer relações com os pares e com os professores por forma a integra-se melhor a nível académico e social; (d) a autoeficácia em ações proativas que diz respeito à perceção da capacidade no aproveitamento de oportunidades que surgem durante a formação, em manter-se atualizado acerca dos conhecimentos referentes à formação e em realizar ações que promovam melhorias na instituição; e, por fim, (e) a autoeficácia na gestão académica que implica a confiança na capacidade de planeamento e envolvimento para cumprir os prazos em relação às atividades académicas. Este conjunto de dimensões cobre as áreas da adaptação académica, assumindo a autoeficácia papel relevante na qualidade dessa adaptação. Sobretudo junto dos estudantes do $1 .^{\circ}$ ano e nas primeiras semanas no ES, a adaptação académica está relacionada com a intenção ou mesmo concretização do abandono (Caprara et al., 2011; Criollo et al., 2017; Tinto, 1975; Yorke \& Longden, 2008).

A par da autoeficácia, outras variáveis pessoais dos estudantes e do seu percurso académico impactam no abandono ou na intenção de abandono dos estudantes do ES (Ambiel et al., 2016; Martin, 2017). A investigação sugere, por exemplo, que as estudantes do sexo feminino tendem a abandonar menos os seus estudos (Peltier, Laden, \& Matranga, 1999) uma vez que apresentam melhores competências de estudo e valorizam mais a educação superior. Num artigo de meta-análise sobre diferenças de sexo na autoeficácia académica, Huang (2013) refere uma superioridade feminina, contudo alerta para a diferenciação dos contextos académicos. As mulheres tendem a apresentar maiores níveis de autoeficácia académica na área das humanidades e das artes, enquanto os homens apresentam níveis mais elevados nas áreas da matemática e computação. $\mathrm{Na}$ área das ciências os dois sexos não se diferenciam.

No entanto, as raparigas abandonam mais os estudos quando estão integradas em cursos e instituições tipicamente masculinos, situação em que, tendencialmente, apresentam menor rendimento académico (OECD, 2008). No caso das mulheres, o abandono pode estar relacionado com dificuldades de integração social nos primeiros tempos no ES (Tinto, 1975) ou, ainda, com dificuldades de gerir o tempo e de conciliação das responsabilidades, profissionais e académicas (Aina, 2013; Stratton, O'Toole, \& Wetzel, 2008). À semelhança de estudos internacionais, um estudo recente em Portugal aponta esta dificuldade de conciliação de papéis por parte das mulheres, nomeadamente na priorização de objetivos e na gestão de tempo (Andrade, van Rhijn, \& Coimbra, 2017).

Relativamente ao percurso escolar prévio, os estudantes com um percurso anterior mais bem-sucedido, com melhores níveis de desempenho e rendimento académico estão mais próximos de alcançar o objetivo de ingressar no curso desejado, sentindo a entrada no ES como um sucesso. Estes estudantes com médias mais altas ingressam em cursos que exigem notas de ingresso mais elevadas e cursos socialmente mais valorizados. Esta vivência contribui para a consolidação de perceções positivas de autoeficácia, tendo assim também impacto positivo na sua perceção de competência, na sua motivação, na formulação de novas metas e objetivos, e no investimento futuro nas tarefas académicas (Azzi \& Polydoro, 2007). Em situação contrária estarão os estudantes que ingressam no ES, com opções vocacionais ajustadas às possibilidades e constrangimentos do sistema de numerus 
clausus, limitando o acesso a cursos e instituições que desejariam (Araújo et al., 2016; Crosling, Heagney, \& Thomas, 2009). Este último grupo de estudantes, partindo de "situação de desconforto" para a sua adaptação académica, mais facilmente experimentarão níveis reduzidos de satisfação, autoeficácia e motivação (Belloc, Maruotti, \& Petrella, 2011). As menores competências académicas destes estudantes no ensino secundário associam-se agora ao menor rendimento no ES, sendo o bom rendimento académico um fator protetor do abandono, aumentando a probabilidade de conclusão do $1 .^{\circ}$ ano com adequado sucesso.

Por último a inexistência de tradição familiar de frequência universitária é um dos aspetos mencionados na literatura impactando nas dificuldades sociais e emocionais. Segundo Dias e Sá (2014), os estudantes de primeira geração apresentam receios mais acentuados relativamente à orientação espacial no campus e insegurança urbana, bem como sentimentos de solidão e dificuldades em lidar autonomamente com a generalidade das situações da vida académica. Estes estudantes, sobretudo quando provenientes de estratos socioeconómicos mais desfavorecidos, tendem a apresentar índices mais baixos de literacia e numeracia, assim como hábitos de estudo e formas de pensamento crítico mais frágeis, afetando negativamente a sua motivação e rendimento académico e aumentando o risco de abandono (Aina, 2013; Stinebrickner \& Stinebrickner, 2014).

Com este estudo pretendemos analisar o impacto das variáveis sexo, média de acesso ao ensino superior, frequência ou não do curso de primeira opção de escolha e o nível de escolaridade dos pais na intenção de abandonar o ES por parte dos estudantes do $1 .^{\circ}$ ano. Esta análise considera também o efeito mediador da autoeficácia, assumindo este globalmente a partir da conjugação das dimensões académica, regulação da formação e interações sociais.

\section{Método}

\section{Participantes}

Participaram no estudo 1085 estudantes do $1 .^{\circ}$ ano de uma universidade pública do norte de Portugal, que ingressaram em cursos do $1 .^{\circ}$ ciclo e em mestrados integrados. $33.7 \%$ dos estudantes são do sexo masculino e $66.4 \%$ do sexo feminino, com idades compreendidas entre os 16 e os 58 anos, com uma média de idade de 18.88 anos $(D P=3.85)$. Finalmente, $43.2 \%$ dos estudantes referem ter ambos os pais com escolaridade ao nível do ensino básico, $31.4 \%$ em que pelo menos um dos pais têm ensino secundário, $14.7 \%$ em que os pais têm pelo menos um elemento com ES, e $10.7 \%$ com ambos os pais com ES. Os estudantes ingressaram em diversos cursos das áreas das ciências humanas, sociais, jurídicas, saúde e engenharia.

\section{Instrumentos}

Questionário Sociodemográfico. Foi administrado um breve questionário sociodemográfico aos estudantes em que se recolheram os dados relativos ao sexo, a média de acesso ao ES (traduzida numa escala de 0 a 20 pontos, exigindo-se 10 pontos para ingressar no ES), a frequência ou não do curso de primeira opção de escolha, o nível educacional dos pais e a existência ou não da intenção de abandonar o ES.

Escala de Autoeficácia na Formação Superior. A recolha das perceções de autoeficácia recorreu à Escala de Autoeficácia na Formação Superior (AEFS) (Vieira, Polydoro, \& Guerreiro-Casanova, 2017). Esta escala avalia três dimensões: (a) autoeficácia académica - definida como a confiança na capacidade de aprender, demonstrar e aplicar o conteúdo do curso (7 itens; e.g.: "Aprender os conteúdos que são necessários à minha formação"); (b) Autoeficácia na regulação da formação - consiste na confiança do estudante na sua capacidade para estabelecer metas, fazer escolhas, planejar, cumprir prazos e autorregular as suas ações no processo de formação (7 itens; e.g.: "Concluir os trabalhos do curso dentro dos prazos estabelecidos"); (c) Autoeficácia na interações sociais - entendida como a confiança na capacidade para se relacionar em termos académicos e sociais com os colegas e com os professores (6 itens; e.g.: "Estabelecer amizades com os colegas do curso").

A escala integra 20 itens face aos quais os estudantes devem indicar o seu nível de confiança através de uma escala tipo Likert de seis pontos, desde (1) Nada confiante até (6) Totalmente confiante. A pontuação na escala total é calculada pela soma das respostas a todos os itens, dividida pelo número total de itens, estando as pontuações superiores associadas a um nível mais elevado de autoeficácia. Neste estudo utilizou-se a versão adaptada e validada para a população portuguesa (Vieira, Polydoro, \& Coimbra, 2009) que apresentou bons valores de consistência interna (alfa de Cronbach) nas três dimensões entre .86 e .90 .

\section{Procedimento}

Recorreu-se a uma amostra de conveniência não probabilística com o critério de inclusão de serem estudantes do $1 .^{\circ}$ ano do ES. A recolha de dados foi efetuada de forma coletiva, no contexto de sala de aula após seis semanas de frequência do ES, com a permissão e colaboração dos docentes das turmas. Seguindo as orientações éticas para a investigação com seres humanos, foram apresentados os objetivos do estudo, mediante os quais os 
estudantes forneceram o seu consentimento escrito para a participação livre e esclarecida, sendo-lhes assegurada a confidencialidade dos dados fornecidos. Procedeu-se à Modelagem de Equações Estruturais tomando na análise do ajustamento do modelo os índices propostos na literatura (Hu \& Bentler, 1999) para os seguintes coeficientes: $\chi^{2} / \mathrm{df}$ (Qui-quadrado por graus de Liberdade), TLI (Índice de Tucker-Lewis), CFI (Índice de ajustamento comparativo) e o RMSEA (raiz da média dos quadrados dos erros de aproximação). Utilizou-se o critério de .05 na significância dos coeficientes de diferenças entre grupos. As análises foram realizadas por recurso ao software estatístico Mplus (versão 7) (Muthén \& Muthén, 2012).

\section{Resultados}

O estudo foi realizado recorrendo a uma amostra de 1085 estudantes do $1 .^{\circ}$ ano do ES, sendo que $125(11.5 \%)$ mencionaram a intenção de abandonar o ES no momento em que estes dados foram recolhidos $\left(6^{\mathrm{a}}-8^{\mathrm{a}}\right.$ semanas após o início das aulas). Na Tabela 1 descrevemos os estudantes diferenciando os resultados de acordo com terem ou não intenção de abandono. De referir que a pontuação global (nota Z) na Escala de Autoeficácia na Formação Académica decorre da variável latente estimada a partir dos resultados nas três dimensões da escala.

Tabela 1

Caracterização dos Estudantes Com e Sem Intenção de Abandono

\begin{tabular}{|c|c|c|c|c|c|c|}
\hline \multirow[t]{2}{*}{ Variáveis } & \multicolumn{3}{|c|}{$\begin{array}{l}\text { Sem intenção de abandono } \\
(n=960)\end{array}$} & \multicolumn{3}{|c|}{$\begin{array}{l}\text { Com intenção de abandono } \\
(n=125)\end{array}$} \\
\hline & $\mathrm{N}$ & & & $n$ & & \\
\hline \multicolumn{7}{|l|}{ Sexo } \\
\hline masculino & 323 & & & 43 & & $4 \%$ \\
\hline feminino & 637 & & & 82 & & $6 \%$ \\
\hline \multicolumn{7}{|l|}{$1^{\mathrm{a}}$ opção curso } \\
\hline $\operatorname{sim}$ & 625 & & & 66 & & \\
\hline não & 335 & & & 59 & & $2 \%$ \\
\hline \multicolumn{7}{|l|}{ Escolaridade dos pais } \\
\hline ambos com ensino básico & 408 & & & 61 & & \\
\hline pelo menos um com ensino secundário & 302 & & & 39 & & \\
\hline pelo menos um com ES & 140 & & & 19 & & \\
\hline \multirow{2}{*}{ ambos com ensino ES } & 110 & & & 6 & & \\
\hline & Min - Max & $M$ & $D P$ & Min-Max & $M$ & $D P$ \\
\hline Média de acesso & $10.20-19.70$ & 15.41 & 1.62 & $11.00-19.10$ & 14.89 & 1.66 \\
\hline Autoeficácia & $-3.17-2.11$ & .049 & .965 & $-3.35-2.11$ & -.408 & 1.18 \\
\hline
\end{tabular}

Comparando os estudantes em função de apresentarem ou não intenção de abandonar o ES, não se verificam diferenças em função do sexo $\left(\chi^{2}=.028, g l=1, p=.87\right)$. No entanto, existe uma diferença estatisticamente significativa em relação ao facto de estarem colocados ou não num curso de primeira opção de escolha $\left(\chi^{2}=7.24, g l=1\right.$, $p<.01)$. Uma taxa superior de estudantes não colocados num curso de primeira opção pensaram em abandonar. Apesar de não se verificar de forma estatisticamente significativa a associação entre a intenção de abandonarem ou não abandonarem com o nível de escolaridade dos pais $\left(\chi^{2}=5.63, g l=3, p=.13\right)$, verifica-se uma taxa superior de estudantes com intenção de abandonar no subgrupo daqueles cujos pais possuem apenas a escolaridade básica.
Analisando a média de acesso ao ES e a pontuação global de autoeficácia, verificam-se diferenças estatisticamente significativas entre os dois grupos de estudantes. A diferença na média de acesso (teste $t=3.37, g l=1.083$, $p<.01)$ traduz que os estudantes com intenção de abandono apresentam uma média mais baixa no acesso ao ES, ao mesmo tempo que apresentam uma média mais baixa na autoeficácia global (teste $t=4.23, g l=10.83$, $p<.001)$.

Face aos objetivos deste estudo, analisamos o impacto das variáveis em presença na intenção de abandono. O modelo testado considerou o impacto das variáveis sexo, média de acesso ao ES, frequência ou não do curso de primeira opção de escolha e o nível de escolaridade 
dos pais na intenção de abandonar, considerando ainda o efeito mediador da autoeficácia (cf. Figura 1). Na análise do ajustamento do modelo consideramos o $\chi 2 / \mathrm{df}$ (Qui-quadrado por graus de Liberdade), TLI (Índice de Tucker-Lewis), CFI (Índice de ajustamento comparativo) e o RMSEA (raiz da média dos quadrados dos erros de aproximação). O presente modelo apresentou $\chi^{2} / \mathrm{df}=2.05$, RMSEA $=0.031$ [0.013 - 0.049], CFI $=0.981, \mathrm{TLI}=0.96$, $\mathrm{SRMR}=0.01$. Como se constata, mesmo não incluindo modificação, o modelo apresenta um bom ajustamento uma vez que apresenta os valores de CFI e TLI acima de .95 e RMSEA abaixo de .06 (Hu \& Bentler, 1999). Analisando os parâmetros estandardizados (cf. Figura 1) podemos verificar que as três dimensões da autoeficácia convergem para um fator geral de Autoeficácia na Formação Superior, aliás com loadings muito altos para cada dimensão (AE aca $\beta=.837, \mathrm{AE}$ regfor $\beta=.962$, $\mathrm{AE}$ intsoc $\beta=.781)$.

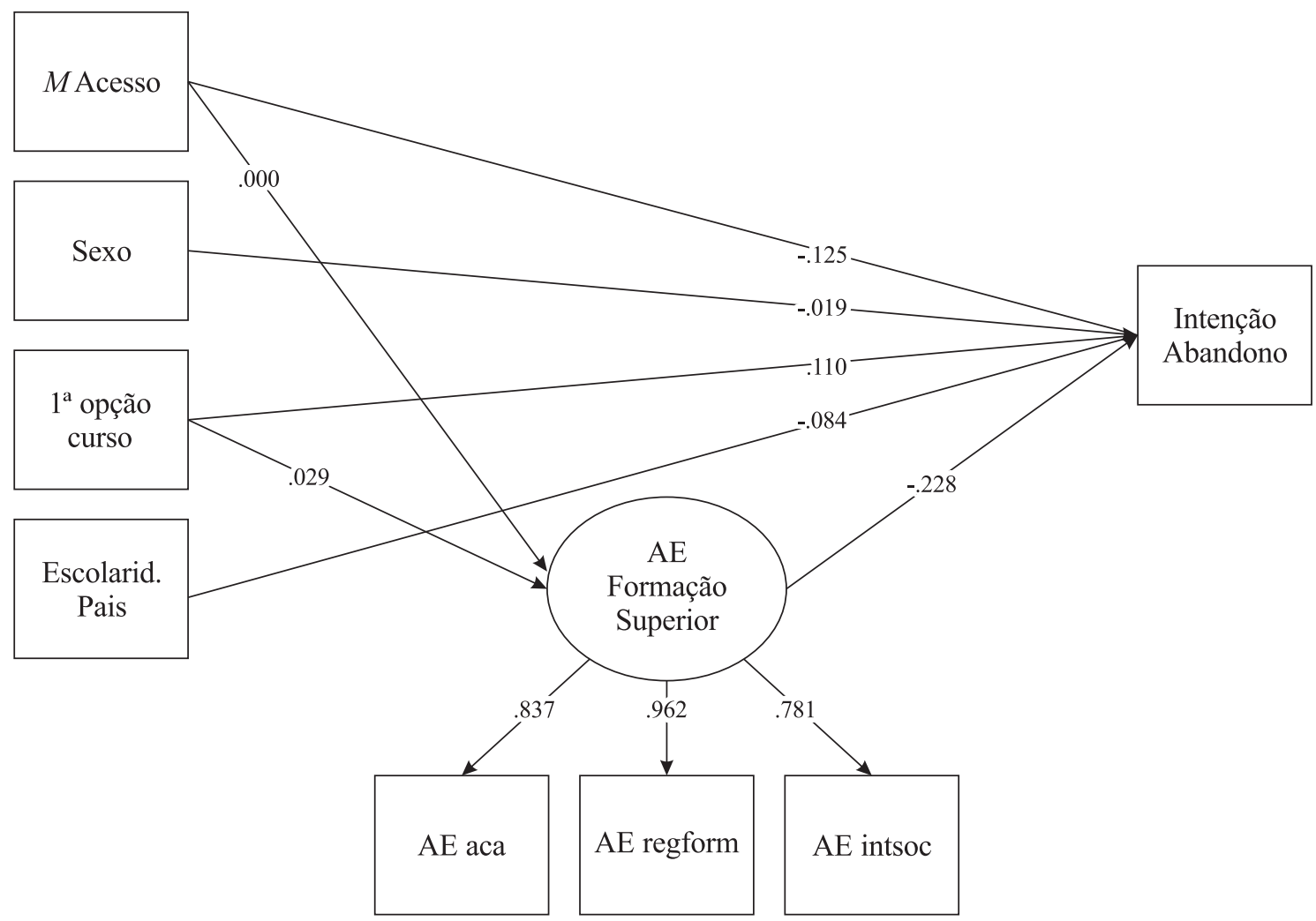

Figura 1. Modelo de Equações Estruturais do Impacto das Variáveis na Intenção de Abandono

Legenda: M Acesso - Média de acesso ao ES; Sexo: 1 - Masculino, 2 - Feminino; $1^{\text {a }}$ opção curso: 1 - Sim, 2 - Não; Escolaridade dos pais: 1 - Ambos com escolaridade básica, 2 - Pelo menos um com ensino secundário, 3 - Pelo menos um com ES, 4 - Ambos com o ES; AE aca - autoeficácia académica, AE regfom - autoeficácia na regulação da formação, AE intsoc - autoeficácia nas interações sociais; Intenção de abandono: 1 - Sim, 2 - Não; Os valores são standardizados e apenas são apresentadas as trajetórias estatisticamente significativas

Tal como é possível verificar na Figura 1, o percurso escolar prévio, expresso na média de acesso ao ES, tem um efeito negativo e estatisticamente significativo na intenção de abandonar a formação superior $(\beta=-.125)$, ou seja, os estudantes com intenção de abandonar o ES tendem a apresentar médias de acesso mais baixas. Ainda que com menor impacto, a média de acesso ao ES tem um impacto significativo na Autoeficácia na Formação Superior, sugerindo que o percurso prévio do estudante tem um efeito direto mais importante na intenção de abandonar o
ES do que na autoeficácia. No entanto, verificamos que a autoeficácia tem uma relação moderada negativa com a intenção de abandonar o curso $(\beta=-.228)$, ou seja, os estudantes com intenção de abandonar o curso apresentam níveis mais baixos de autoeficácia.

O sexo dos estudantes também está relacionado com a intenção de abandonar o ES ( $\beta=-.019)$, mas não está relacionado com os níveis de autoeficácia. Os estudantes do sexo masculino apresentam taxa superior da intenção de abandono. O mesmo acontece com as habilitações 
dos pais, que têm uma relação negativa com a intenção de abandono, ou seja, uma taxa superior de intenção de abandonar o ES ocorre nos estudantes provenientes de famílias com menor escolaridade. Por último, frequentar o curso de primeira opção é também importante para a intenção de abandono. Os estudantes que têm intenção de abandonar tendencialmente não frequentam um curso de primeira opção $(\beta=-.019)$.

\section{Considerações Finais}

O ingresso no ES constitui um período desafiante para os estudantes, em muitos casos com dificuldades assinaláveis, quer em termos pessoais quer sociais e académicas. Para alguns estudantes a vivência de um novo contexto, com atividades, tarefas e responsabilidades tão diferenciadas e desconhecidas, representa desafios e exigências, potenciando o insucesso académico e abandono do ES. Esta situação explica, aliás, o maior número de abandonos em Portugal no decurso do $1 .^{\circ}$ ano, sugerindo maior atenção das instituições às condições de acolhimento dos seus novos estudantes.

Confrontando os dados obtidos no presente estudo com outros estudos referenciados na literatura podemos apontar algumas convergências. O percurso escolar prévio, assim como a frequência de um curso de primeira opção de escolha, têm impacto na intenção de abandonar o ES por parte dos estudantes, nomeadamente já no decurso do $1 .^{\circ}$ ano. Os estudantes com médias de acesso mais baixas, e automaticamente entrando em cursos menos valorizados socialmente e que não são sua primeira escolha, apresentam com mais frequência a intenção de abandonar o ES (Azzi \& Polydoro, 2007; Casanova et al., 2017). Por sua vez, o efeito direto da média de acesso na autoeficácia é menos relevante, sugerindo que o percurso prévio do estudante tem um efeito direto mais importante na intenção de abandonar do que na autoeficácia. $\mathrm{O}$ fato dos estudantes que têm intenção de abandonar tendencialmente não frequentarem o curso de primeira opção de escolha alerta as instituições para o impacto do $n u$ merus clausus nas intenções de abandono do ES logo no decurso do $1 .^{\circ}$ ano.

Os resultados obtidos apontam que estudantes do sexo masculino apresentam maior intenção de abandonar o ES (Huang, 2013; Peltier et al., 1999), não havendo diferenças de sexo nos níveis de autoeficácia avaliada. Também as habilitações dos pais estão relacionadas com a taxa de ocorrência da intenção de abandono. Estudantes cujos pais possuem apenas a escolaridade básica apresentam uma taxa superior de intenção de abandono (Aina,
2013; Stinebrickner \& Stinebrickner, 2014), e de novo aqui não se encontra uma relação entre níveis de escolaridade dos pais e de autoeficácia.

Por sua vez, e como seria expectável, também a autoeficácia impacta na intenção de abandono (Ambiel et al., 2016; Azzi \& Polydoro, 2007). Assim, o grupo de estudantes com intenção de abandonar o curso apresenta menores níveis de autoeficácia. Isto se explica pelo efeito mediador das perceções de autoeficácia nas vivências académicas, em particular no que respeita à fixação de objetivos de aprendizagem e níveis de rendimento ou no enfrentamento das dificuldades na adaptação e superação dos níveis de ansiedade associados a tais dificuldades (Ambiel, Santos, \& Dalbosco, 2016; Azzi \& Polydoro, 2007; Casanova et al., 2017; Soares et al., 2015). Os autores apontam que, estando a autoeficácia associado ao rendimento académico, acaba também por estar associada às taxas de abandono em virtude do rendimento impactar na persistência ou abandono dos estudantes (Bernardo et al., 2015).

Como grande conclusão deste estudo, sendo as taxas de abandono mais frequentes no $1 .^{\circ}$ ano, e estando o percurso académico anterior e as perceções de autoeficácia associadas à sua ocorrência, importaria que as instituições implementassem alguns programas de apoio aos seus novos estudantes, por exemplo desenvolvendo competências de estudo e autorregulação da aprendizagem, ajudando os estudantes a explorar novas oportunidades de projetos de carreira em função do curso que frequentam, e reforçando o sentimento de pertença à instituição e ao curso ou desenvolvimento da sua autonomia e estratégias de confronto com as dificuldades sentidas na sua adaptação ao ES (Carvalho \& Taveira, 2012).

A terminar, algumas limitações se podem apresentar ao presente estudo, podendo converter-se em pistas para futuras pesquisas na área. Assim, seria pertinente a inclusão de outras variáveis relevantes na explicação do abandono, em particular as relacionadas com os projetos vocacionais e o desenvolvimento de carreira. Por outro lado, seria importante neste tipo de estudos ir a outros contextos fora da sala de aula para constituir a amostra e, desta forma, aumentar a probabilidade de incluir estudantes que possam não estar a frequentar as aulas. Ainda, dever-se-ia aprofundar o impacto no abandono da frequência pelos estudantes de um curso que não foi primeira escolha tomando separadamente as áreas científicas dos cursos pois pode o fenómeno do abandono e as intenções de abandonar assumirem formas diversas de acordo com esta variável. 


\section{Referências}

Aina, C. (2013). Parental background and university dropout in Italy. Higher Education, 65(4), 437-456. http://doi. org/10.1007/s10734-012-9554-z

Ambiel, R. A. M., Santos, A.A. A., \& Dalbosco, S. N. P. (2016). Motivos para evasão, vivências acadêmicas e adaptabilidade de carreira em universitários. Psico, 47(4), 288. http://doi.org/10.15448/1980-8623.2016.4.23872

Andrade, C., van Rhijn, T., \& Coimbra, S. (2017). Gender Differences in School-Family Conflict and School-Family Enrichment in Nontraditional Portuguese Students. The Journal of Continuing Higher Education, 65(1), 13-24. http://doi.org/10.1080/07377363.2017.1272439

Araújo, A. M., Santos, A. A., Noronha, A. P., Zanon, C., Ferreira, J. A., Casanova, J. R., \& Almeida, L. S. (2016). Dificuldades antecipadas de adaptação ao Ensino Superior: Um estudo com alunos do primeiro ano. Revista de Estudios e Investigación en Psicología Y Educación, 3(2), 102-111. http://doi.org/10.17979/reipe.2016.3.2.1846

Astin, A. W. (1984). Student Involvement : A Developmental Theory for Higher Education. Journal of College Student Development, 25(4), 297-308. http://doi.org/10.1016/0263-

Azzi, R. G., \& Polydoro, S. (2007). Auto-eficácia em diferentes contextos. Campinas, São Paulo: Alínea.

Bandura, A. (1996). Social cognitive theory of human development. In T. Husen \& T. N. Postlethwaite (Eds.), International Encyclopedia of Education (2nd ed., pp. 5513-5518). Oxford: Pergamin Press.

Bean, J. P. (1980). Dropouts and turnover: The synthesis and test of a causal model of student attrition. Research in Higher Education, 12(2), 155-187. doi: 10.1007/BF00976194

Bean, J. P., \& Metzner, B. S. (1985). A conceptual model of nontraditional undergraduate student attrition. Review of Educational Research, 55(4), 485-540.

Belloc, F., Maruotti, A., \& Petrella, L. (2011). How individual characteristics affect university students drop-out: A semiparametric mixed-effects model for an Italian case study. Journal of Applied Statistics, 38(10), 2225-2239. http://doi.org/10.1080/02664763.2010.545373

Bernardo, G. A. B., Cerezo Menéndez, R., Rodríguez-Muñíz, L. J., Núñez Pérez, J. C., Tuero Herrero, E., \& Esteban, G. M. (2015). Predicción del abandono universtirario: Variables explicativas y medidas de prevención. Revista Fuentes, 63-84. http://doi.org/http://dx.doi.org/10.12795/revistafuentes.2015.i16.03

Bresó, E., Schaufeli, W. B., \& Salanova, M. (2011). Can a self-efficacy-based intervention decrease burnout, increase engagement, and enhance performance? A quasi-experimental study. Higher Education, 61(4), 339-355. http://doi.org/10.1007/s10734-010-9334-6

Carvalho, M., \& Taveira, M. C. (2012). A implementação de decisões vocacionais: Revisão da literatura. Revista Brasileira de Orientação Profissional, 13(1), 27-35.

Casanova, J. R., Esteban, M., Cervero, A., Bernardo, A., \& Almeida, L. S. (2017). Questões vocacionais e aprendizagem no abandono precoce do Ensino Superior. In A. P. Marques, C. Sá, J. R. Casanova, \& L. S. Almeida (Eds.), Ser diplomado do Ensino Superior: Escolhas, percursos e retornos (pp. 175-190). Braga: CIEd, Universidade do Minho.

Criollo, M., Romero, M., \& Fontaines-Ruiz, T. (2017). Autoeficacia para el aprendizaje de la investigación en estudiantes universitarios. Psicología Educativa, 23(1), 63-72. http://doi.org/10.1016/j.pse.2016.09.002

Crosling, G., Heagney, M., \& Thomas, L. (2009). Improving student retention in Higher Education: Improving teaching and learning. Australian Universities' Review, 51(2), 9-18.

Dias, D., \& Sá, M. J. (2014). The impact of the transition to HE: Emotions, feelings and sensations. European Journal of Education, 49(2), 291-303. http://doi.org/10.1111/ejed.12058

Duque, L. C., Duque, J. C., \& Suriñach, J. (2013). Learning outcomes and dropout intentions: An analytical model for Spanish universities. Educational Studies, 39(3), 261-284. http://doi.org/10.1080/03055698.2012.724353

Fredricks, J. A., Blumenfeld, P. C., \& Paris, A. H. (2004). School engagement: Potential of the concept, state of the evidence. Review of Educational Research, 74(1), 59-109. http://doi.org/10.3102/00346543074001059

Guerreiro-Casanova, D. C., \& Polydoro, S. A. J. (2011). Autoeficácia e integração ao ensino superior: um estudo com estudantes de primeiro ano. Psicologia: Teoria E ..., 13(1), 75-88. Retrieved from http://pepsic.bvsalud.org/scielo. php?pid=S1516-36872011000100006\&script=sci_arttext

Hu, L., \& Bentler, P. M. (1999). Cutoff criteria for fit indexes in covariance structure analysis: Conventional criteria versus new alternatives. Structural Equation Modeling: A Multidisciplinary Journal, 6(1), 1-55. http://doi. org/10.1080/10705519909540118 
Huang, C. (2013). Gender differences in academic self-efficacy: A meta-analysis. European Journal of Psychology of Education, 28(1), 1-35. http://doi.org/10.1007/s10212-011-0097-y

Martin, J. M. (2017). It just didn't work out: Examining nonreturning students' stories about their freshman experience. Journal of College Student Retention: Research, Theory and Practice, 19(2), 176-198. http://doi. org/10.1177/1521025115611670

Muthén, L., \& Muthén, B. (2012). Mplus user's guide (version 7.0). Mplus User's Guide (Seventh Edition), 1-850. http:// doi.org/10.1111/j.1600-0447.2011.01711.x

Nunes, M. F. O. (2008). Funcionamento e desenvolvimento das crenças de auto-eficácia: Uma revisão. Revista Brasileira de Orientação Profissional, 9(1), 29-42.

OECD. (2008). Higher education to 2030. Paris: Retrieved from http://www.keepeek.com/Digital-Asset-Management/ oecd/education/higher-education-to-2030-volume-1-demography_9789264040663-en\#.Wmr-CK51-4Q\#page1

Pascarella, E. T., \& Terenzini, P. T. (1991). How college affects students. Jossey-Bass Publishers.

Peltier, G. L., Laden, R., \& Matranga, M. (1999). Student persistence in college: A review of research. Journal of College Student Retention: Research, Theory and Practice, 1(4), 357-375. http://doi.org/10.2190/L4F7-4EF5-G2F1-Y8R3

Polydoro, S. A., \& Guerreiro-Casanova, D. C. (2010). Escala de Autoeficácia na Formação Superior: Construção e estudo de validação. Avaliação Psicológica, 9(2), 267-278.

Soares, A. M., Pinheiro, M. do R., Manuel, J., \& Canavarro, J. M. P. (2015). Transição e adaptação ao Ensino Superior e a demanda pelo sucesso nas instituições portuguesas. Psychologica, 58(2). http://doi.org/10.14195/1647-8606_58

Spady, W. G. (1970). Dropouts from higher education: An interdisciplinary review and synthesis. Interchange, 1(1), 64-85. http://doi.org/10.1007/BF02214313

Stinebrickner, R., \& Stinebrickner, T. (2014). Academic performance and college dropout: Using longitudinal expectations data to estimate a learning model. Journal of Labor Economics, 32(3), 601-644. http://doi.org/10.1086/675308

Stratton, L. S., O’Toole, D. M., \& Wetzel, J. N. (2008). A multinomial logit model of college stopout and dropout behavior. Economics of Education Review, 27(3), 319-331. http://doi.org/10.1016/j.econedurev.2007.04.003

Tinto, V. (1975). Dropout from Higher Education: A Theoretical synthesis of recent research. Review of Educational Research, 45(1), 89-125. http://doi.org/10.3102/00346543045001089

Vieira, D. A., Polydoro, S., \& Coimbra, J. L. (2009). Auto-eficácia na formação superior (AEFS): Estudo de validação em estudantes do ensino superior em Portugal. In XIV Conferência Internacional de Avaliação Psicológica: Formas e Contextos (pp. 63-64). Campinas, São Paulo: Universidade de São Francisco.

Vieira, D. A., Polydoro, S., \& Guerreiro-Casanova, D. C. (2017). Escala de Autoeficácia na Formação Superior (AEFS). In L. S. Almeida, M. R. Simões, \& M. M. Gonçalves (Eds.), Adaptação, desenvolvimento e sucesso académico dos estudantes do Ensino Superior: Instrumentos de avaliação (pp. 111-123). Braga: ADIPSIEDUC.

Recebido 30/01/2018

Reformulado 19/06/2018

Aceite final 07/10/2018

Sobre os autores

Joana R. Casanova é Doutoranda em Ciências da Educação, Especialidade de Psicologia da Educação, no Instituto de Educação, Universidade do Minho, Portugal, com financiamento da Fundação para a Ciência e Tecnologia (SFRH/ $\mathrm{BD} / 117902 / 2016)$.

Antonio Cervero Fernandez-Castañon é Doutorando em Psicologia na Faculdade de Psicologia da Universidade de Oviedo, Espanha, com financiamento do Governo do Principado das Astúrias através do programa Severo Ochoa de ajudas pre-doutorais para a investigação e docência (ref. BP16014).

José Carlos Nuñez Pérez é Professor Catedrático na área de Psicologia Evolutiva e da Educação na Faculdade de Psicologia da Universidade de Oviedo, Espanha.

Ana B. Bernardo Gutiérrez é Professora Contratada Doctora (acreditada como titular de universidade) na área de Psicologia Evolutiva e da Educação na Faculdade de Psicologia da Universidade de Oviedo, Espanha.

Leandro S. Almeida é Professor Catedrático de Psicologia da Educação no Instituto de Educação da Universidade do Minho, Portugal. 